\title{
Multiple Exciton Generation and Dynamics in InP/CdS Colloidal Quantum Dots
}

DOI:

10.1021/acs.jpcc.6b11744

\section{Document Version}

Accepted author manuscript

Link to publication record in Manchester Research Explorer

\section{Citation for published version (APA):}

Smith, C., Leontiadou, M., Clark, P., Lydon, C., Savjani, N., Spencer, B., Flavell, W., O'Brien, P., \& Binks, D. (2017). Multiple Exciton Generation and Dynamics in InP/CdS Colloidal Quantum Dots. Journal of Physical Chemistry C, 121(4). https://doi.org/10.1021/acs.jpcc.6b11744

\section{Published in:}

Journal of Physical Chemistry C

\section{Citing this paper}

Please note that where the full-text provided on Manchester Research Explorer is the Author Accepted Manuscript or Proof version this may differ from the final Published version. If citing, it is advised that you check and use the publisher's definitive version.

\section{General rights}

Copyright and moral rights for the publications made accessible in the Research Explorer are retained by the authors and/or other copyright owners and it is a condition of accessing publications that users recognise and abide by the legal requirements associated with these rights.

\section{Takedown policy}

If you believe that this document breaches copyright please refer to the University of Manchester's Takedown Procedures [http://man.ac.uk/04Y6Bo] or contact uml.scholarlycommunications@manchester.ac.uk providing relevant details, so we can investigate your claim.

\section{OPEN ACCESS}


This document is confidential and is proprietary to the American Chemical Society and its authors. Do not copy or disclose without written permission. If you have received this item in error, notify the sender and delete all copies.

\section{Multiple Exciton Generation and Dynamics in InP/CdS Colloidal Quantum Dots}

\begin{tabular}{|r|l|}
\hline Journal: & The Journal of Physical Chemistry \\
\hline Manuscript ID & jp-2016-11744f.R1 \\
\hline Manuscript Type: & Article \\
\hline Date Submitted by the Author: & 04-Jan-2017 \\
\hline Complete List of Authors: & $\begin{array}{l}\text { Smith, Charles; University of Manchester, School of Physics and Astronomy } \\
\text { \& Photon Science Institute } \\
\text { Clark, Pip; University of Manchester, School of Physics and Astronomy \& } \\
\text { Photon Science Institute } \\
\text { Leontiadou, Marina A.; University of Manchester, School of Physics and } \\
\text { Astronomy \& Photon Science Institute } \\
\text { Lydon, Claire; University of Manchester, School of Chemistry } \\
\text { Savjani, Nicky; The University of Manchester, School of Chemistry; Nanoco } \\
\text { Technologies Ltd. } \\
\text { Spencer, Ben; The University of Manchester, School of Materials } \\
\text { Flavell, Wendy; University of Manchester, School of Physics and Astronomy } \\
\text { \& Photon Science Institute } \\
\text { O'Brien, Paul; The University of Manchester, School of Chemistry } \\
\text { Binks, David; University of Manchester, School of Physics and Astronomy \& } \\
\text { Photon Science Institute }\end{array}$ \\
\hline
\end{tabular}




\title{
Multiple Exciton Generation and Dynamics in InP/CdS Colloidal Quantum Dots
}

Charles T. Smith ${ }^{1}$, Marina A. Leontiadou ${ }^{1}$, Pip C. J. Clark ${ }^{1}$, Claire Lydon ${ }^{2}$, Nicky Savjani ${ }^{2 \dagger}$, Ben F. Spencer ${ }^{3}$, Wendy R. Flavell ${ }^{1}$, Paul O'Brien ${ }^{2}$, and David J. Binks ${ }^{1 *}$.

${ }^{1}$ School of Physics and Astronomy \& Photon Science Institute, University of Manchester, Manchester, M13 9PL, UK.

${ }^{2}$ School of Chemistry, University of Manchester, Manchester, M13 9PL, UK.

$†$ Nanoco Technologies Ltd, 46 Grafton Street, Manchester, M13 9NT, UK

${ }^{3}$ School of Materials, University of Manchester, Manchester, M13 9PL, UK.

*E-mail: david.binks@manchester.ac.uk

\begin{abstract}
We report measurements of multiple exciton generation and recombination in $\mathrm{InP} / \mathrm{CdS}$ (core/shell) colloidal quantum dots. Ultrafast transient absorption spectroscopy was used to measure the sub-nanosecond charge dynamics for a range of pump fluences and for different pump photon energies. Analysis of the resulting transients was used to determine the quantum yield of multiple exciton generation, which was found to be $1.22 \pm 0.01$ for a pump photon energy equivalent to three times the band gap.
\end{abstract}




\section{INTRODUCTION}

Colloidal quantum dots (CQDs) are nanometer-scale semiconductor crystals that can be produced by facile solution-phase chemistry. ${ }^{1}$ The size-tunable band gap, $E_{g}$, of CQDs coupled with the low-cost methods by which they can be fabricated and processed makes them attractive for a number of different applications. These include acting as the luminophores in efficient light sources, ${ }^{2}$ and as the photo-absorbing species in image processing devices ${ }^{3}$ and third-generation solar cells. ${ }^{1,4}$ CQDs have the potential to increase the efficiency of solar cells by the process of multiple exciton generation (MEG), also known as carrier multiplication. As first described by Shockley and Queisser, ${ }^{5}$ the efficiency of a conventional single-junction solar cell under unconcentrated sunlight is limited to $\sim 33 \%$, largely by the cooling to the band edge of carriers generated by the absorption of a photon with energy in excess of $E_{g}$. However, for CQDs the process of MEG can become competitive with carrier cooling, so that some or all of the initial carrier kinetic energy is used to generate additional electron-hole pairs rather than being lost as heat. Including MEG in the Shockley and Queisser analysis shows that this effect has the potential to increase the maximum solar cell efficiency to $44 \%{ }^{6}$

MEG in CQDs was first proposed in $2001^{7}$ and first experimentally demonstrated in 2005 for PbSe CQDs. ${ }^{8}$ Since then MEG has been demonstrated in CQDs composed of a range of single materials, including $\mathrm{PbS},{ }^{9} \mathrm{HgTe},{ }^{10} \mathrm{CdHgTe}^{11}$ and $\mathrm{CuInSe}_{2},{ }^{12}$ as well as in other forms of colloidal nanostructures such as $\mathrm{PbSe}$ nanorods ${ }^{13}$ and $\mathrm{PbS}$ nanosheets ${ }^{14}$. One of the most useful aspects of the solution-phase synthesis of CQDs is that it allows a core-shell structure to be readily produced by the growth of a shell or shells of different material(s) around the original CQD. A spherical heterostructure is thus produced allowing control of the wave function of photo-generated carriers, and thus of their interactions and dynamics, and this can be used to 
engineer the properties of CQDs for MEG-enhanced solar cells. These heterostructures can be in several forms depending on the relative $E_{g}$ and band offsets of the core and shell materials, including: Type I, in which both band edge carriers are confined to the core; Type II, where one carrier is confined to the core and the other to the shell; and quasi-Type II, where one or other of carriers is confined to the core or shell, and the other is delocalised over the whole CQD. A Type I CQD design can be used to reduce the trapping of hot carriers by the surface states that compete with MEG. A study of dispersions of $\mathrm{InP} / \mathrm{ZnS} / \mathrm{ZnO}$ (core/shell/shell) Type I CQDs reported an efficiency of $0.3,{ }^{15}$ defined as the rate of increase with photon energy (in units of $E_{g}$ ) of the number of excitons produced per absorbed photon. However, a later study of CQDsensitized solar cells ${ }^{16}$ showed that the addition of such wide band gap outer shell to CQDs can result in significantly reduced photovoltaic efficiency, an effect attributed to the outer shell acting as a barrier to charge extraction from the CQDs. In contrast, the shell is not a barrier to extraction for one or both of the photo-generated charges in a Type II or quasi-Type II CQD design; moreover, this design can also enhance the efficiency of the MEG process itself. An upper limit to MEG efficiency of 0.5 was estimated for Type II CdTe/CdSe CQDs dispersions ${ }^{17}$ and this high efficiency was attributed to a reduction in the wave function overlap between carriers resulting in a decreased rate of Auger cooling, another process that competes with MEG. A quasi-Type II PbSe/CdSe CQD design was used to achieve an MEG efficiency of 0.3, by suppressing the hole cooling which competes with MEG in this system. ${ }^{13}$

In this work, we study MEG in dispersions of quasi-Type II CQDs formed by the growth of a CdS shell around a InP core; in this CQD structure, the electron is delocalised over the core and shell whilst the hole is confined to the InP core, ${ }^{18}$ as illustrated in Figure 1. InP CQDs form with a cubic crystal structure (lattice constant $5.87 \AA)^{19}$ but the thermodynamically stable phase 
of $\mathrm{CdS}$ is hexagonal ${ }^{18}$. However, CdS can also exist in a cubic phase (lattice constant $\left.5.83 \AA\right)^{19}$ which, as we show below, can form when thin shells of CdS grow on to an InP core. Thus, InP/CdS CQDs can be produced with a common crystal structure and a lattice mismatch of only $0.7 \%$; in contrast, to grow a CdS/InP CQD with a similar common crystal structure would be more problematic since it would require the initial synthesis of $\mathrm{CdS}$ cores in the thermodynamically unstable cubic phase. There are several reasons why InP/CdS CQDs are of particular interest for MEG-enhanced solar cells. As mentioned above, Type I CQDs based on InP cores exhibit good MEG efficiency. ${ }^{15}$ The addition of a CdS shell can redshift the absorption edge significantly, ${ }^{18}$ so that it closer to the ideal value for optimal exploitation of the solar spectrum. ${ }^{5}$ A CdS shell has also been shown to be an effective means of passivating surface traps, as evidenced by the increase in photoluminescence (PL) and reduction in PL intermittency (also known as 'blinking') resulting from the addition of such a shell. ${ }^{18}$ Moreover, this surface passivation does not come at the cost of extraction efficiency from the CQD. Wu et al. ${ }^{20}$ showed that the characteristic electron transfer time from an InP CQD to a methylviologen acceptor molecule was just $11 \mathrm{ps}$, and was little changed after the addition of a CdS shell at $15 \mathrm{ps}$. Importantly, both of these values are much less than the biexciton lifetime in InP/CdS CQDs, which can be as long as $7 \mathrm{~ns},{ }^{18}$ indicating that the additional excitons produced by MEG can be extracted with high efficiency from the CQD rather than being lost to recombination. The single exciton recombination lifetime is also extended to several hundred nanoseconds in $\mathrm{InP} / \mathrm{CdS}$ CQDs, for both isolated CQDs ${ }^{18}$ and CQD-acceptor complexes; ${ }^{18}$ calculations for similar $\mathrm{CdSe} / \mathrm{CdTe} \mathrm{CQDs}$ indicate that the increase in recombination lifetime produced by a quasi-Type II structure can significantly increase solar cell efficiency. ${ }^{21}$ However, it is still unclear how MEG efficiency itself will be affected by the addition of a CdS shell to an InP CQD, which will 
depend on the interplay of several competing processes, each of which is affected by the change in structure. The reduced wave function overlap between carriers produced by the quasi-Type II structure may simultaneously reduce the rate of MEG and of the Auger relaxation with which it competes, but by different amounts. The reduced confinement resulting from the addition of the $\mathrm{CdS}$ will reduce the separation of energy levels for the electron, which may increase the rate of phonon-mediated cooling. ${ }^{22}$

The efficiency of MEG in InP/CdS CQDs is reported for the first time in this work. The characterisation of these CQDs by transmission electron microscopy (TEM), X-ray diffraction (XRD), X-ray photoelectron spectroscopy (XPS), and absorption and photoluminescence (PL) spectroscopies is described initially. The sub-nanosecond charge dynamics are then studied using ultrafast transient absorption spectroscopy. The absorption transients are obtained for a range of pump fluences, and for pump photon energies both below and above the MEG threshold, and analysed to obtain the MEG quantum yield, QY. We thus show that the MEG QY for quasi-Type II InP/CdS CQDs is similar to that already reported for Type I InP CQDs. This establishes that changing the CQD heterostructure in such a way does not adversely affect the MEG efficiency, enabling the advantages of a quasi-Type II design for a CQD-based solar cell, such as increased excitonic lifetimes and reduced band gap, to be exploited without reducing the potential benefit to photo-voltaic efficiency produced by MEG. 


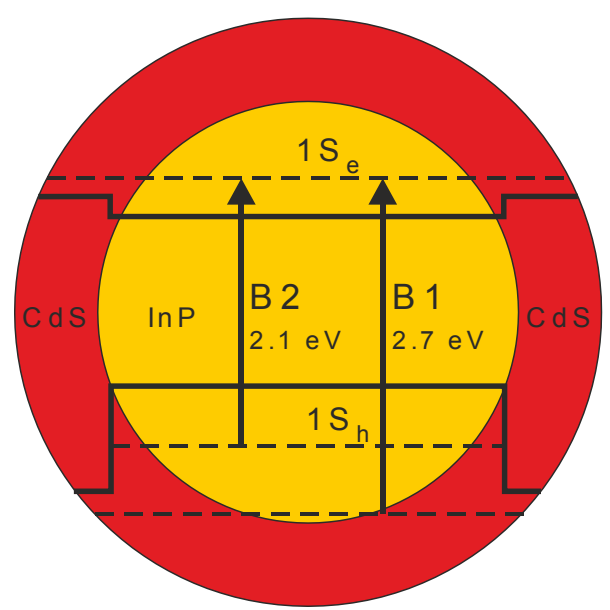

Figure 1. Schematic representation of the InP/CdS (core/shell) CQD structure and the resulting quasi-Type II band alignment. ${ }^{20}$ Also shown is the lowest electron energy level in the conduction band and the hole energy levels corresponding to the B1 and B2 transitions, with indicative energies corresponding to the samples studied.

\section{RESULTS AND DISCUSSION \\ CHARACTERISATION}

Steady-state absorption and PL spectra for the InP/CdS CQDs are shown in Figure 2. The absorption spectrum of the InP cores before the addition of the CdS shell is also shown for comparison. The InP cores have an absorption edge at $550 \mathrm{~nm}$, which corresponds to an average CQD diameter of $3.9 \mathrm{~nm}^{23}$ The average diameter of $20 \mathrm{InP} / \mathrm{CdS}$ CQDs measured from the TEM image shown in Figure 3 was found to be $5.2 \pm 0.1 \mathrm{~nm}$, with a standard deviation of $0.6 \mathrm{~nm}$, indicating the shell thickness is approximately $0.7 \mathrm{~nm}$. On the addition of the CdS shell, the absorption edge is red-shifted to $600 \mathrm{~nm}$ and weakens; the corresponding transition is shown in Figure 1, labelled as B2. Both observations are consistent with the formation of a quasi-Type II CQD, and are attributable to a decreased confinement of the electron as its wave function spreads into the shell, resulting in reduced wave function overlap between the carriers, which reduces the absorption strength of the band edge transition. The absorbance of the InP/CdS CQDs increases 
sharply for wavelengths less than $\sim 500 \mathrm{~nm}$; this corresponds to transitions now involving delocalised hole as well as de-localised electron states with consequently increased wave function overlap and thus absorption strength. The lowest energy transition of this type is to the conduction band minimum from a valence band state somewhat below the maximum, and is labelled as B1 in Figure 1, (this labelling corresponds to bleach peaks observed in previous transient absorption studies ${ }^{20}$ ).

No significant PL was obtained from the InP cores, but after the addition of the shell emission increased and the photoluminescence quantum yield was measured to be $10 \pm 2 \%$; this can be attributed to the CdS acting as a barrier that reduces the interaction of holes with the CQD surface, which has been shown to mediate non-radiative recombination. ${ }^{24,25}$ The PL spectrum has a peak at $700 \mathrm{~nm}$ and a half-width at half-maximum on the short wavelength side of $50 \mathrm{~nm}^{26}$ These optical properties are consistent with previous results both for InP/CdS CQDs ${ }^{18,20}$ and other quasi-Type II CQDs. $^{27}$

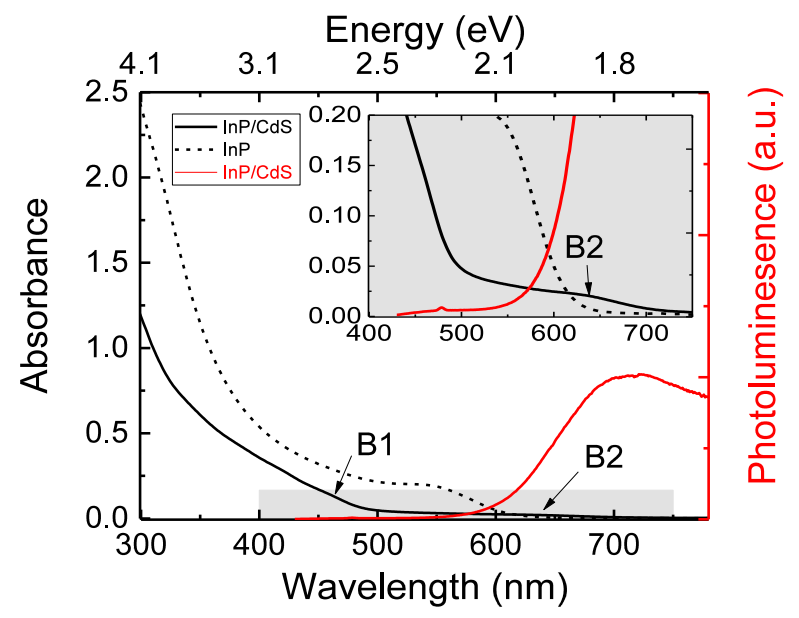

Figure 2. Absorbance (black) and photoluminescence (red) spectra for the InP (dashed lines) and InP/CdS (solid lines) CQD samples. Inset: an expanded view of the absorption edge region. The labelled absorption features, B1 and B2, correspond to the transitions illustrated by Figure 1. 
Also shown in Figure 3 are the X-ray diffraction patterns for the InP and InP/CdS CQDs, with reference peaks corresponding to the cubic phases of both $\mathrm{InP}$ and $\mathrm{CdS}$ also shown for comparison. The diffraction peaks observed for the InP CQDs agree well with the expected peak positions and relative intensities. Dennis et al. ${ }^{18}$ reported that for 'giant' $\mathrm{InP} / \mathrm{CdS} \mathrm{CQDs}$, with shells that were up to $4 \mathrm{~nm}$ thick, the CdS forms in its more thermodynamically stable hexagonal phase. However, here the diffraction peaks are little changed on the addition of the shell, and are consistent with the formation of $\mathrm{CdS}$ in its cubic phase (zinc blende/Hawleyite). This indicates that for thin shells the cubic template provided by the InP is a more important factor than thermodynamic stability in determining the lattice structure of the shell.
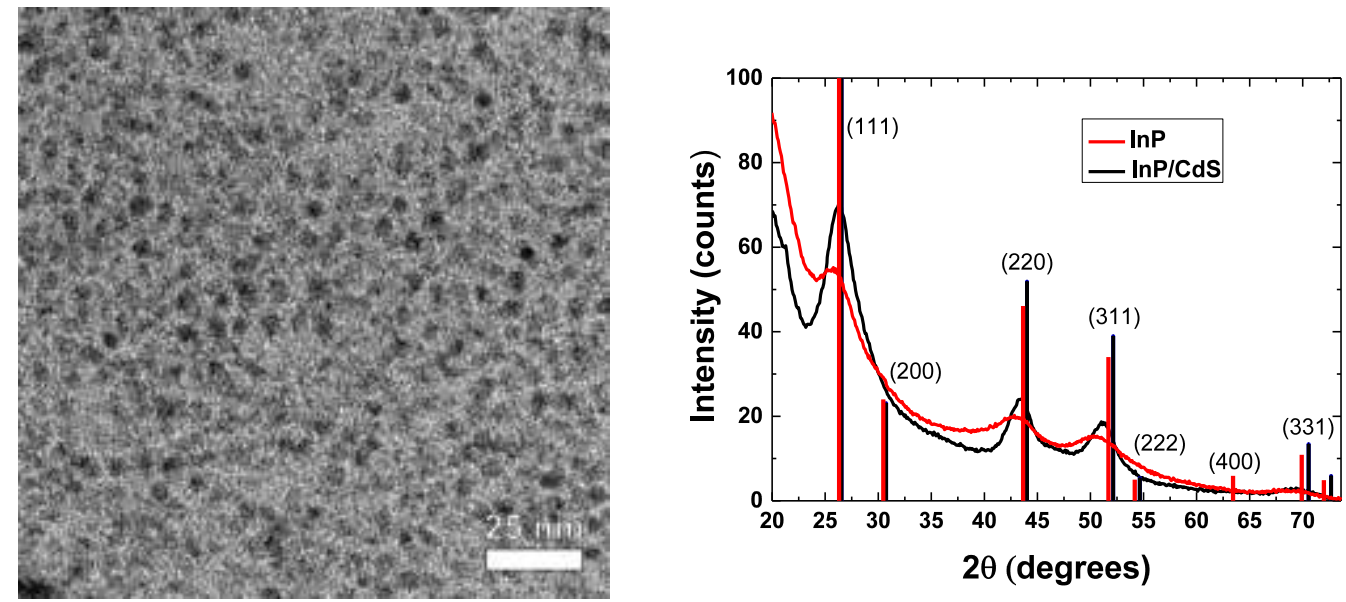

Figure 3. (Left) TEM image of the InP/CdS CQDs. (Right) X-ray diffraction patterns for the InP (red) and InP/CdS (black) CQDs. Reference peaks for InP (red) and cubic phase (zinc blende/Hawleyite) CdS (black) are also shown (Powder Diffraction Files 01-073-1983 and 00-042-1411, respectively).

X-ray photoelectron spectroscopy (XPS) was used to investigate the composition of the InP and InP/CdS CQDs. As described in the experimental section, the ligands on the as-synthesised CQDs were exchanged for 3-mercaptopropionic acid (3-MPA) in order to prevent charging during exposure to the X-ray beam. The normalised In 3d, P 2p, Cd 3d, S 2p, C 1s and O 1s core peaks are presented in Figure 4 for both the InP core and the InP/CdS core/shell CQDs. The 
cores showed a P:In ratio of $0.4 \pm 0.1$. This ratio was not significantly different after the addition of the CdS at $0.5 \pm 0.1$, indicating that the core composition is not appreciably changed by the shelling process. The low value of the P:In ratio determined by XPS measurements has been reported previously ${ }^{28,29}$ for InP CQDs synthesised by the same method and was attributed to the effect of an In rich surface. In Figure 4(b) the lower binding energy (BE) P 2p doublet (P 2p $\mathrm{p}_{3 / 2}$ at $128.9 \mathrm{eV})$ is characteristic of $\mathrm{InP}$. The second, higher $\mathrm{BE}$ doublet $\left(\mathrm{P} 2 \mathrm{p}_{3 / 2}\right.$ at $\left.133.3 \mathrm{eV}\right)$ is attributed to $\operatorname{InPO}_{x}$. The same degree of oxidation of the core can be seen both before and after the addition of the shell, implying that no further oxidation of the core occurs during the shelling procedure. Similar observations have been reported previously for InP CQDs and were explained as arising due to a ketonization reaction during synthesis producing an oxidative environment which results in conversion of the top layer of the InP CQDs into $\operatorname{InPO}_{x}$ (where $x=3,4$, but predominantly 4). ${ }^{29}$ However, the authors noted further oxidation on the addition of a ZnS shell, which is not observed here for the case of a CdS shell. ${ }^{28,29}$

Observation of the Cd 3d and S 2p signals, shown in Figures 4 (c) and (d), revealed that Cd and S are present in broadly equal amounts with $\mathrm{Cd}$ :In and $\mathrm{S}(\mathrm{CdS}): \mathrm{In}$ ratios of $2.6 \pm 0.3$ and $3.2 \pm 0.5$ respectively, consistent with the addition of a CdS shell to the InP core. Two S $2 p$ doublets were observed in the $\mathrm{InP} / \mathrm{CdS}$ sample, one characteristic of $\mathrm{CdS}$ at $161.7 \mathrm{eV} \mathrm{BE},{ }^{30}$ and a second at 162.9 eV BE attributed to the 3-MPA ligand bound to the CQD surface. The latter was also observed in the InP sample, which was also capped with 3-MPA. No S $2 p$ doublets were observed at higher binding energies (centered around $168 \mathrm{eV} \mathrm{BE}$ ), where sulfur oxidation products such as $\mathrm{SO}_{4}{ }^{2-}$ and $\mathrm{SO}_{3}{ }^{2-}$ would be present, ${ }^{31}$ indicating that the shell did not oxidize. The $\mathrm{Cd} 3 \mathrm{~d}_{5 / 2}$ peak was observed at $405.2 \mathrm{eV} \mathrm{BE}$, consistent with other reports for $\mathrm{CdS}^{32} \mathrm{~A}$ 
binding energy shift was observed in the In $3 \mathrm{~d}$ peaks between the InP/CdS and InP CQDs (see Figure 4(a)), with In $3 \mathrm{~d}_{5 / 2}$ was found at $445.0 \mathrm{eV} \mathrm{BE}$ and $445.3 \mathrm{eV}$ BE respectively.

The C 1s spectra, (see Figure 4(e)) contain a dominant peak associated with hydrocarbon chains, at $284.8 \mathrm{eV}$, and two higher binding energy peaks at $286.2 \mathrm{eV}$ and $288.5 \mathrm{eV}$, associated with C-S and $\mathrm{O}-\mathrm{C}=\mathrm{O}$ respectively, originating from the $3-\mathrm{MPA}$ ligands. The $\mathrm{O} 1$ s spectra (see Figure 4(f)) contain two components in both samples. The lower binding energy component at $531.4 \mathrm{eV}$ is attributed to hydroxide species ( $\mathrm{InOOH}$ or $\left.\mathrm{In}(\mathrm{OH})_{3}\right)$, and the higher component at 532.5 is attributed to both $\mathrm{InPO}_{x}$ and $\mathrm{O}-\mathrm{C}=\mathrm{O} .^{29}$ 


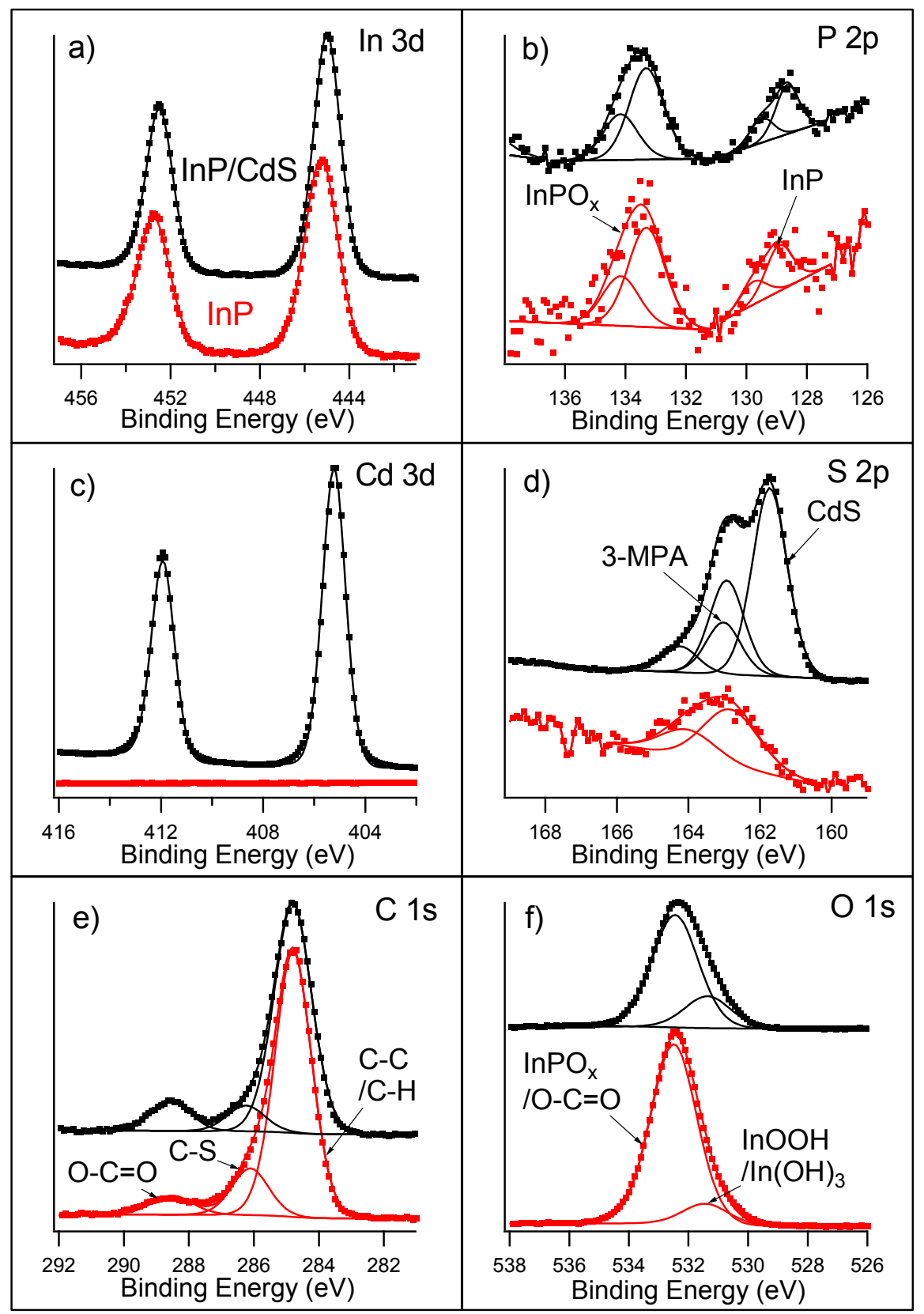

Figure 4. Comparison of (a) In 3d, (b) P 2p, (c) Cd 3d (d) S 2p, (e) C 1s, and (f) O 1s X-ray photoelectron spectra for an InP (bottom, red) and InP/CdS (top, black) CQDs. The spectra are normalised to the In 3d signal for each sample.

The Cd 3d:In 3d XPS ratio was used to calculate the shell thickness of the CdS Shell with an analytical model developed by Shard. ${ }^{33}$ This method also requires the core size, determined as $3.9 \mathrm{~nm}$ from the absorption spectrum, and the inelastic mean free paths of electrons in the core 
and shell material, calculated with the TPP-2M formula. ${ }^{34-36}$ This calculation revealed a thickness of $0.7 \pm 0.1 \mathrm{~nm}$, approximately equal to three monolayers of $\mathrm{CdS},{ }^{37}$ and thus gave total diameter of $5.2 \pm 0.2 \mathrm{~nm}$, consistent with the size extracted from TEM imaging of the core/shell CQD.

\section{ULTRAFAST CHARGE DYNAMICS}

Figure 5 shows the fractional transmittance change spectrum for the InP/CdS CQDs acquired at a pump probe time delay of $3 \mathrm{ps}$, corresponding to the time of maximum bleach signal. Two distinct bleach peaks are evident, corresponding to the B1 and B2 transitions, and are both attributed to state-filling at the conduction band edge. The inset to Figure 5 compares the decay dynamics of both features, which were found to occur with identical rates, as has been observed previously for InP/CdS CQDs ${ }^{20}$ and in other quasi-Type II CQDs, ${ }^{27}$ confirming this attribution. Both these bleach features thus allow the transient population of photogenerated band-edge electrons to be monitored; for this study the B1 bleach was used primarily, due to its larger response and thus better signal-to-noise ratio.

The evolution of the B1 bleach for a range pump fluences, $J$, is shown in Figure 6 for pump wavelengths of $420 \mathrm{~nm}$ and $232 \mathrm{~nm}$, corresponding to excitation photon energies of $2.96 \mathrm{eV}$ and $5.35 \mathrm{eV}$, respectively. Taking the peak of the PL as a measure of $E_{g}$, these photon energies are equivalent to $1.7 E_{g}$ and $3.0 E_{g}$ and thus correspond to pumping below and above the threshold for MEG, respectively. Each transient exhibits a very rapid initial rise of a few ps duration followed by a decay to a plateau. This behavior is typical for CQDs, ${ }^{38}$ with the rise corresponding to increasing state-filling as photo-generated electrons cool to the band edge, and the decay corresponding to the recombination of biexcitons, formed either by MEG or by the absorption of more than one photon per CQD per excitation pulse, which depopulates the band 
edge by an efficient Auger process; the plateau is associated with state-filling by single excitons which recombine with a lifetime that is long compared to the duration of the experiment. Charges can become trapped on the surface of some CQD types for a time longer than the duration between pump pulses. This can result in the formation of a trion when an exciton created by the absorption of a subsequent pump photon combines with the geminate partner of the trapped charge. These trions decay on a similar time-scale to biexcitons, and their presence can lead to an over-estimation of the MEG QY if they are mis-identified. ${ }^{39}$ However, it has also been shown ${ }^{40,41}$ that sufficient stirring or flowing of the sample can prevent trion formation by refreshing the pumped sample volume. Transients obtained when the sample was static and when it was stirred are compared in Figure S1 of the Supporting Information. There is no significant difference between these transients, indicating that trion formation is not an important process for the InP/CdS CQD samples under the experimental conditions used here.

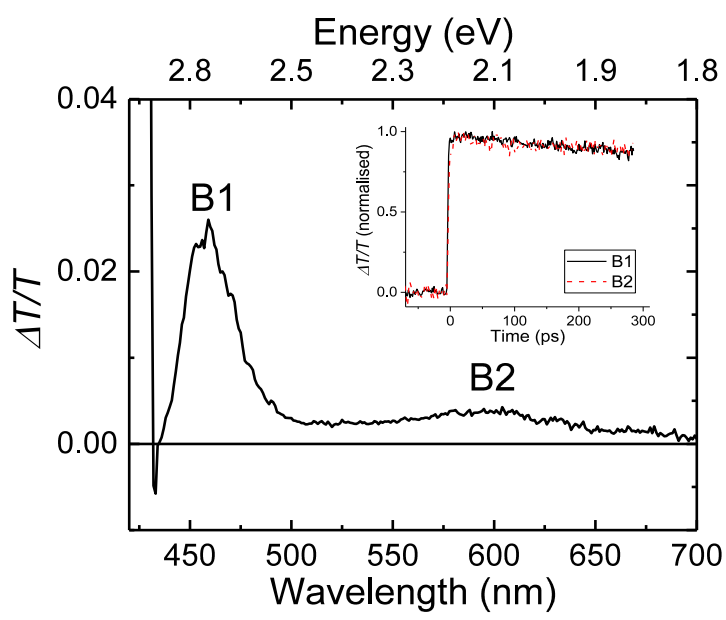

Figure 5. Fractional transmittance change, $\Delta T / T$, spectrum for the InP/CdS CQD sample collected at a pump-probe delay of $\sim 3 \mathrm{ps}$. The sample was pumped at a wavelength of $420 \mathrm{~nm}$ with a fluence of $1.1 \times 10^{14}$ photons $\mathrm{cm}^{-2}$ per pulse. The large feature for wavelengths less than $\sim 430 \mathrm{~nm}$ is due to scattered pump light. 

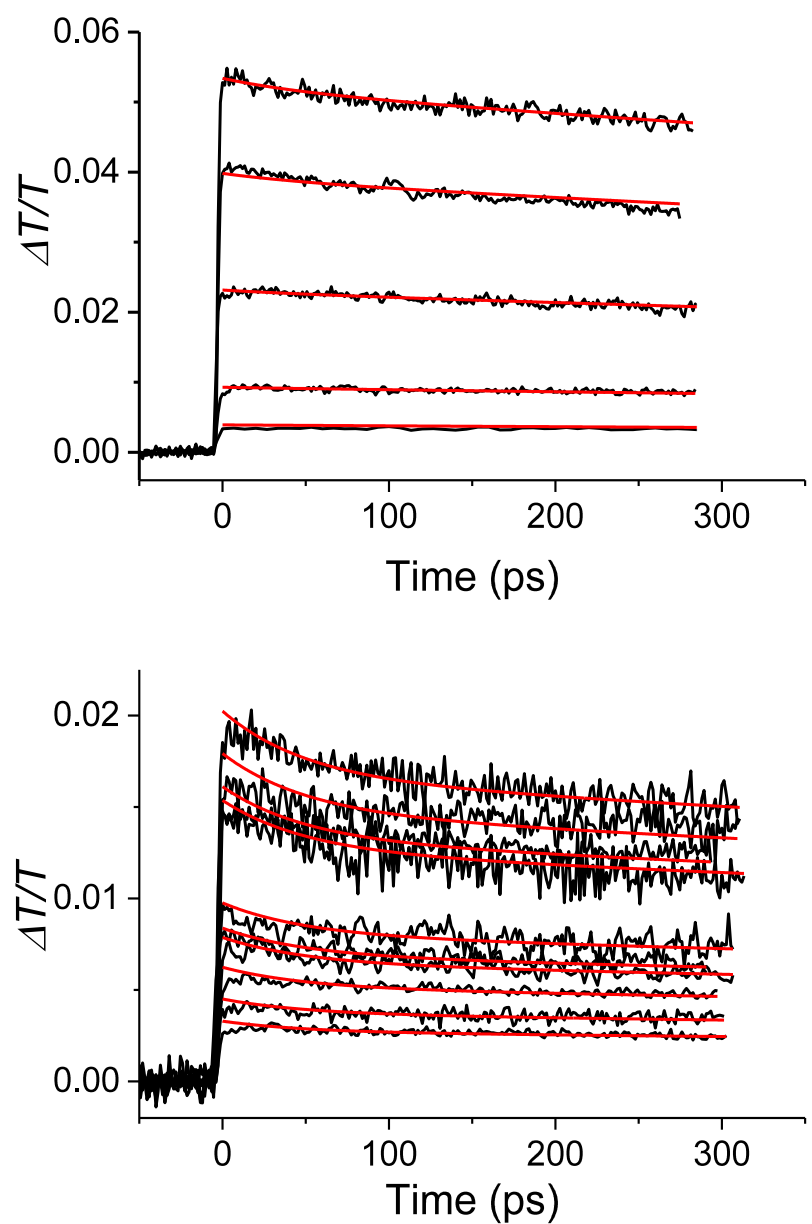

Figure 6. Fractional transmittance change $(\Delta T / T)$ transients for the peak of the $\mathrm{B} 1$ bleach for the $\mathrm{InP} / \mathrm{CdS}$ CQD sample below and above the MEG threshold. The sample was pumped at a wavelength of (top panel) $420 \mathrm{~nm}$ with fluences of $(0.5-3) \times 10^{14}$ photons $\mathrm{cm}^{-2}$ per pulse, and (bottom panel) $232 \mathrm{~nm}$ with fluences of $(0.4-4.8) \times 10^{14}$ photons $\mathrm{cm}^{-2}$. Red lines are fits using Equation 2.

The ratio, $R$, between the bleach amplitude at its peak and at the plateau depends on the average number of excitons per photo-excited CQD, with $R>1$ corresponding to the formation of multi-excitons in some fraction of the CQD population. ${ }^{42}$ At high pump fluences, multiexcitons can be created in a CQD by the absorption of more than one pump photon per pulse, as well as by MEG. However, for low pump fluences, the probability of absorbing more than one pump photon per pulse becomes negligible and in this case an observation of $R>1$ indicates 
that MEG is occurring. For core only and Type I core/shell CQDs, the MEG QY for a particular pump photon energy is typically determined from similar bleach transients by measuring $R$ for a range of fluences and extrapolating to the value corresponding to negligible fluence by fitting these data to the following equation ${ }^{42}$

$$
R(J)=\delta Q Y\langle N\rangle[1-\exp (-\langle N\rangle)]^{-1}
$$

where $\langle N\rangle=\sigma J$ is the average number of photons absorbed per CQD, $\sigma$ is the absorption cross section at the pump wavelength, and $\delta$ is a factor which accounts for the decay of single excitons over the observed time window. However, Eqn. (1) is only valid for samples with an absorbance length, $l_{A}$, for the pump wavelength which is long compared to the beam path through the sample, $l_{S} \cdot{ }^{42}$ Moreover, as discussed previously, ${ }^{10}$ for $l_{A} \ll l_{S}$ the value of $\Delta T / T$ observed will be reduced, since the volume of excited sample through which the probe beam passes is decreased as the pump beam is completely absorbed close to the front facet of the cuvette. A dilemma arises because the magnitude of $\Delta T / T$ is also proportional to the absorbance at the probe wavelength, $\mathrm{A}$, i.e. reducing the sample concentration so that $l_{A}$ is long compared to $l_{S}$ results in a weak fractional change in transmittance.

The InP/CdS CQDs studied here is just such a case for which $l_{A} \ll l_{S}$; as shown in Figure 2, the pump absorbance, $A_{p}$, at the shortest wavelength of $232 \mathrm{~nm}$ is $\sim 40$ times greater than at the probe wavelength of $470 \mathrm{~nm}$. Therefore, we analyse the effect of MEG on the fractional transmittance change transients, $\Delta T(t) / T$, and thereby determine its $Q Y$, by instead fitting them to the following expression (derived in the Supporting Information) which takes into account strong pump absorbance. For an average number of photons absorbed per CQD as the pump beam enters the sample, $\langle N\rangle_{0}$, 


$$
\frac{\Delta T(t)}{T}=\frac{A}{A_{p}}\left[D(t) \sum_{n=1}^{\infty} \frac{\left(-\langle N\rangle_{0}\right)^{n}}{n \cdot n !}\left(e^{-n A_{p}}-1\right)-E(t)\left(e^{-\langle N\rangle_{0} e^{-A_{p}}}-e^{-\langle N\rangle_{0}}\right)\right]
$$

where

$$
\begin{gathered}
D(t)=\frac{k_{X X}+k_{X}}{2 k_{X X}} e^{-k_{X} t}-\frac{k_{X}-k_{X X}}{2 k_{X X}} e^{-\left(k_{X X}+k_{X}\right) t} \\
E(t)=(2-Q Y)\left(\frac{k_{X}}{2 k_{X X}} e^{-k_{X} t}-\frac{k_{X}-k_{X X}}{2 k_{X X}} e^{-\left(k_{X}+k_{X X}\right) t}\right)
\end{gathered}
$$

and where $k_{X X}$ and $k_{X}$ are the rate constants for decay of biexcitons and single excitons, respectively. A global fit of Eqn. (2) to the transients shown in Figure 6 was undertaken for both pump wavelengths simultaneously. The values of $A_{p}$ and $A$ determined from the steady-state absorbance spectrum, i.e. Figure 2, were used, whilst values of $k_{X}$ and $k_{X X}$ common to all the transients were determined by the fitting process. The value of $Q Y$ was fixed to unity for those transients corresponding to pumping below the threshold for MEG; a $Q Y$ value for pumping at a photon energy of $3 E_{g}$, common to the corresponding transients, was found from the fit.

This process yielded biexciton and single exciton lifetimes of $\tau_{X X}=k_{X X}^{-1}=50 \pm 2$ ps and $\tau_{X}=k_{X}^{-1}=3.05 \pm 0.06 \mathrm{~ns}$, respectively. This biexciton lifetime is consistent with the value of $58 \pm 12$ ps for a similar InP/CdS CQDs reported previously for excitation below the MEG threshold. ${ }^{18}$ It is also somewhat larger than the $41 \pm 3$ ps biexciton lifetime reported for an $\mathrm{InP} / \mathrm{ZnS} / \mathrm{ZnO}$ (core/shell/shell) Type I CQDs with a similar $E_{g}$, consistent with the reduced wave function overlap in a quasi-Type II CQD. Moreover, at $50 \mathrm{ps}$ this lifetime is significantly longer than the characteristic time for electron transfer to an acceptor molecule reported by $\mathrm{Wu}$ et al. ${ }^{20}$ suggesting that extraction of the additional charge carriers generated by MEG should be possible with good efficiency in this system. The single exciton lifetime measured is also consistent with 
the observations of $\mathrm{Wu}$ et al. ${ }^{20}$ of $\sim 4 \mathrm{~ns}$, determined from measurement of PL decay transients. A similar measurement on the current sample yielded a decay that was well-described over a $10 \mathrm{~ns}$ time-window by a lifetime of $4 \pm 1 \mathrm{~ns}-$ see Figure S3 in Supporting Information.

The MEG $Q Y$ for pumping at a photon energy equivalent to $3 E_{g}$ was determined to be $1.22 \pm 0.01$ by the fitting process. For InP/ZnS/ZnO Type I CQDs with a similar $E_{g}$ an MEG $Q Y$ of $1.18 \pm 0.03$ for a photon energy equivalent to $2.6 E_{g}$ has been previously reported. ${ }^{15}$ However, that work used the position of the absorption edge as a measure of $E_{g}$, rather than the PL peak as in this study; using instead the PL peak, yields a photon energy equivalent to $2.7 E_{g}$. For its reported efficiency of 0.3 , the Type I InP/ZnS/ZnO CQDs would thus be expected to have a MEG $Q Y$ of $1.27 \pm 0.04$ for a pump photon energy equal to $3 E_{g}$. The efficiency of the MEG process in CQDs with an InP core thus seems to be not greatly affected by the change from a Type I to a quasi-Type II core/shell heterostructure, with the difference in MEG $Q Y$ at $3.0 E_{g}$ reported for the two types being similar to the experimental accuracy.

\section{SUMMARY AND CONCLUSION}

InP/CdS CQDs have been synthesised and characterised by absorbance and photoluminescence spectroscopy, transmission electron microscopy, X-ray diffraction, and X-ray photoelectron spectroscopy. These measurements show that quasi-Type II core/shell structures have been synthesized, with a zinc blende crystal phase. The core diameter was determined to be $3.9 \pm 0.1 \mathrm{~nm}$ from optical measurements and the thickness of the shell was $0.7 \mathrm{~nm}$, as determined consistently by both TEM and XPS. An oxide layer was found to form on the surface of the Inrich InP core, but not on the surface of the CdS shell layer, which was broadly stoichiometric. The multi-exciton dynamics in these CQDs have been investigated on a sub-nanosecond time- 
scale using ultrafast transient absorption spectroscopy. The bleach transients produced by biexciton recombination were analysed, taking into account the effect of strong pump absorbance, and were used to determine the quantum yield of multiple exciton generation in $\mathrm{InP} / \mathrm{CdS} \mathrm{CQDs}$ for these first time. This was found to be similar to that previously reported for Type I InP CQDs.

An MEG quantum yield not adversely affected by a change in CQD structure from Type I to quasi-Type II allows the advantages of a quasi-Type II design to be exploited in CQD-based solar cells without sacrificing the potential benefit of MEG. The extended biexciton and single exciton lifetimes confirmed in this study and resulting from the reduced wave-function overlap between charge carriers produced by the quasi-Type II structure, allow more time for charge extraction before recombination. In addition to forming a quasi-Type II structure, the CdS shell also effectively passivates the InP core surface, but without adding a barrier to electron extraction. Finally, the CdS shell also redshifts the band gap towards the optimum for exploitation of the solar spectrum. These advantages will be key to the effective exploitation of multiple exciton generation for enhanced solar cell performance.

\section{METHODS}

\section{Synthesis}

$\mathrm{InP} / \mathrm{CdS}$ nanoparticles were synthesised according to literature procedures, with slight modifications. ${ }^{18,20}$ The amount of CdS precursor solution added was calculated based on the InP core size, itself calculated from the absorption spectrum, described in the literature. ${ }^{23}$

Indium acetate $\left(\mathrm{InAc}_{3}, 0.117 \mathrm{~g}, 0.4 \mathrm{mmol}\right)$, myristic acid (MA, $\left.0.274 \mathrm{~g}, 1.2 \mathrm{mmol}\right)$ and $14 \mathrm{~mL}$ of dry 1-octadecene (ODE) were degassed and kept under vacuum for 30 minutes, followed by 
heating to $120^{\circ} \mathrm{C}$ under vacuum for a further 30 minutes. The mixture was then heated to $285^{\circ} \mathrm{C}$ under argon, and tristrimethylsilyl phosphine (TMSP, $1 \mathrm{ml}, 0.2 \mathrm{mmol}$ ) was injected and the temperature dropped to $270{ }^{\circ} \mathrm{C}$ for the growth period. The reaction was stopped after 4 minutes by removing the heat source and cooled to room temperature. The InP QDs were precipitated out of solution by the addition of ethanol. This was repeated several times and the washed QDs were re-dispersed in dry toluene for analysis.

Precursor solutions of cadmium oleate and sulfur were prepared for the shelling process. The cadmium solution $(0.04 \mathrm{M})$ was prepared by dissolving $\mathrm{CdO}(0.077 \mathrm{~g}, 0.60 \mathrm{mmol} 1)$ in $\mathrm{OA}$ $(1.2 \mathrm{~mL})$ and $15 \mathrm{~mL}$ of dry $\mathrm{ODE}$ at $300{ }^{\circ} \mathrm{C}$ to obtain a colourless solution. The precursor solution was then maintained at above $100^{\circ} \mathrm{C}$. A sulfur injection solution $(0.015 \mathrm{M})$ was prepared by dissolving sulfur (20 mg $0.62 \mathrm{mmol})$ in $20 \mathrm{~mL}$ of ODE at $100{ }^{\circ} \mathrm{C} .5 \mathrm{~mL}$ of oleylamine and $3 \mathrm{~mL}$ of ODE were degassed. Washed cores in hexane $(3.7 \mathrm{~mL}, \sim 44 \mu \mathrm{M})$ were added and the solvent subsequently removed under vacuum with the temperature gradually raised to $100^{\circ} \mathrm{C}$. The flask was refilled with Ar and the temperature was raised to $150^{\circ} \mathrm{C}$ for the first cation addition. (Note: the first addition of cadmium oleate and the initial annealing step were conducted at $150{ }^{\circ} \mathrm{C}$ due to an observed sensitivity of the InP core to etching at high temperatures under these reaction conditions, i.e. in presence of oleylamine and/or with these particular shell precursors). The cadmium precursor solution was added dropwise to the solution, followed by 10 minutes' annealing and the first injection of the sulfur solution. The temperature was then raised to $240{ }^{\circ} \mathrm{C}$ for all subsequent precursor additions. The first shell was annealed for 30 minutes at this elevated temperature. All subsequent additions were annealed at this temperature at for 45 minutes. Three layers of CdS shell were formed in the course of this reaction. After the shelling was complete, the reaction was cooled to room temperature and washed by precipitating with 
ethanol. The washed samples were re-dispersed in dry hexane or toluene for the optical studies in this report, and diluted to an absorbance of approximately 0.1 at the band edge transition wavelength. Samples were kept under nitrogen after washing and for all the optical studies.

\section{X-ray diffraction}

X-ray diffraction measurements were made on a Bruker D8 Discover diffractometer, equipped with a LynxEYE detector and a Cu source $(\lambda=1.5406 \AA ̊)$ with generator settings of $30 \mathrm{~mA}$ and, $35 \mathrm{kV}$. Data were collected in continuous scan mode, over the $2 \theta$ range $10-75^{\circ}$, with step size 0.1 and step time $411 \mathrm{~s}$. The samples were prepared by repeated washing in ethanol and redissolution in dry hexane. The hexane solution was dropped onto a glass slide in multiple layers and dried before measurement.

\section{Transmission electron microscopy}

Bright field images were obtained using a Philips CM20 transmission electron microscope equipped with a $\mathrm{LaB}_{6}$ electron source and operated at $200 \mathrm{kV}$. The samples were prepared by repeated washing with ethanol and re-dissolution in dry hexane. The dilute hexane solution was dropped onto holey carbon TEM grids, washed with dry hexane and dried under vacuum at room temperature before use.

\section{X-ray photoelectron spectroscopy}

The CQDs $(3 \mathrm{ml})$ were precipitated with ethanol and re-dispersed in chloroform $(3 \mathrm{~mL})$ before being added to a solution of 3-mercaptopropionic acid (3ml, $0.03 \mathrm{~mol})$ in $\mathrm{H} 2 \mathrm{O}(3 \mathrm{~mL})$ with stirring under argon. The mixture was stirred for 2 hours, until the dots had moved into the aqueous layer. They were then washed alternately with toluene and ethanol (3 times with each). 
The CQDs were re-dispersed in methanol then drop cast onto glass and XPS was performed with a Kratos Axis Ultra spectrometer, with excitation from focused monochromated Al $\mathrm{K} \alpha$ (1486.6 eV) X-rays and using an electron flood gun for charge neutralization. Peaks were recorded with a pass energy of $40 \mathrm{eV}$. All spectra were calibrated to $\mathrm{C} 1 \mathrm{~s}$ hydrocarbon at $284.8 \mathrm{eV}$. Peaks were fitted with a nonlinear Shirley-type background, using 70\% Gaussian 30\% Lorentzian line shapes. Core level intensities were corrected for their photoionization cross sections $^{43}$ and relative sampling depths.

\section{Optical studies}

The steady state absorption and the PL spectra were measured with the use of a Perkin Elmer Lambda 1050 UV/Vis/NIR spectrometer and a Horiba Jobin Yvon Fluorolog model iHR(FL322) spectrofluorometer, respectively. The PL quantum yield measurements were made with the same spectrofluorometer, utilising its integrating sphere attachment and an excitation wavelength of $420 \mathrm{~nm}$.

The sub-nanosecond charge dynamics were studied using a home-built ultrafast transient absorption spectrometer which has been reported previously. ${ }^{10,11,15,25} \mathrm{~A}$ Ti:Sapphire regenerative amplifier (Spectra-Physics Spitfire-Pro) seeded by a mode-locked Ti:Sapphire oscillator (Spectra-Physics Tsunami) produces pulses of $100 \mathrm{fs}$ duration and $\sim 1 \mathrm{~mJ}$ energy at a wavelength of $\sim 800 \mathrm{~nm}$ and $1 \mathrm{kHz}$ repetition rate. Using a beam splitter, 95\% of this beam is passed to an optical parametric amplifier (OPA, Light Conversion Ltd. TOPAS-C) with harmonic generating crystals to produce the pump beam, tuneable from the infra-red to the ultra-violet with a minimum wavelength of $232 \mathrm{~nm}$. The pump beam is passed through a mechanical chopper synchronised to half the pulse repetition rate and then focused onto the sample. A neutral density 
filter is used to tune the time-averaged pump power at the sample. At $420 \mathrm{~nm}$, with $1 \mathrm{~mW}$ output and a beam diameter of $2.2 \mathrm{~mm}$, the pump density was $26 \mathrm{~mW} \mathrm{~cm}{ }^{-2}$, which is equivalent to a fluence of $1.1 \times 10^{14}$ photons $\mathrm{cm}^{-2}$ per pulse. At $232 \mathrm{~nm}$ with a similar beam diameter, the maximum fluence was $0.5 \times 10^{14}$ photons $\mathrm{cm}^{-2}$ per pulse. The remaining $5 \%$ of the beam from the amplifier is directed through a computer-controlled delay stage to vary the arrival time difference between pump and probe pulses at the sample. This beam is then passed through a sapphire plate to produce a white light continuum, which is split to form the probe and reference beams. The first was directed through the sample and then both through a spectrometer (Princeton Instruments Acton sp2500i) and detected by Si photodiodes. The changes of the probe beam were recorded using a digital lock-in amplifier (Stanford Research Systems SR830). Data shown here are averages of 5-10 scans. In order to prevent photo-charging of the CQDs ${ }^{15}$ the samples were stirred by a magnetic stirring system (Thermo Scientific Variomag Mini) at approximately $1000 \mathrm{rpm}$.

The photoluminescence lifetimes were measured using the time correlated single photon counting technique. ${ }^{44,45}$ A mode-locked Ti:sapphire laser (Mai Tai HP, Spectra-Physics) was used to produce $\sim 100 \mathrm{fs}$ pulses at $80 \mathrm{MHz}$ repetition rate and a wavelength of $840 \mathrm{~nm}$. This rate was reduced to $2 \mathrm{MHz}$ by an acousto-optic pulse picker (Pulse Select, APE) before the wavelength was converted to $420 \mathrm{~nm}$ via second harmonic generation (APE Harmonic Generator) for sample excitation. The resultant emission was passed through a monochromator (Spex 1870c) tuned to the PL peak before detection by a multi-channel plate (Hamamatsu R3809U-50). The time correlation of the detected photons was performed using a TCC900 PC card from Edinburgh Instruments. 


\section{SUPPORTING INFORMATION}

Comparison of stirred and static fractional transmittance change data for InP/CdS CQD.

Derivation of equation 2. Photoluminescence decay curve for InP/CdS CQD.

\section{ACKNOWLEDGEMENTS}

This work was funded by the EPSRC under Grant No. EP/K008544/1.

\section{REFERENCES}

(1) Kim, J. Y.; Voznyy, O.; Zhitomirsky, D.; Sargent, E. H., 25th Anniversary Article: Colloidal Quantum Dot Materials and Devices: A Quarter-Century of Advances. Adv. Mater. 2013, 25, 4986-5010.

(2) Shirasaki, Y.; Supran, G. J.; Bawendi, M. G.; Bulovic, V., Emergence of Colloidal Quantum-Dot Light-Emitting Technologies. Nat Photon 2013, 7, 13-23.

(3) Aslam, F.; Stevenson-Hill, J.; Binks, D. J.; Daniels, S.; Pickett, N. L.; O'Brien, P., Effect of Nanoparticle Composition on the Performance of Photorefractive Polymers. Chem. Phys. 2007, 334, 45-52.

(4) Du, J., et al., Zn-Cu-in-Se Quantum Dot Solar Cells with a Certified Power Conversion Efficiency of 11.6\%. J. Am. Chem. Soc. 2016, 138, 4201-4209.

(5) Shockley, W.; Queisser, H. J., Detailed Balance Limit of Efficiency of P-N Junction Solar Cells. J. Appl. Phys. 1961, 32, 510-\&.

(6) Hanna, M. C.; Nozik, A. J., Solar Conversion Efficiency of Photovoltaic and Photoelectrolysis Cells with Carrier Multiplication Absorbers. J. Appl. Phys. 2006, 100.

(7) Nozik, A. J., Spectroscopy and Hot Electron Relaxation Dynamics in Semiconductor Quantum Wells and Quantum Dots. Annu. Rev. Phys. Chem. 2001, 52, 193-231.

(8) Schaller, R. D.; Klimov, V. I., High Efficiency Carrier Multiplication in Pbse Nanocrystals: Implications for Solar Energy Conversion. Phys. Rev. Lett. 2004, 92, 186601.

(9) Binks, D. J., Multiple Exciton Generation in Nanocrystal Quantum Dots - Controversy, Current Status and Future Prospects. PCCP 2011, 13, 12693-12704.

(10) Al-Otaify, A.; Kershaw, S. V.; Gupta, S.; Rogach, A. L.; Allan, G.; Delerue, C.; Binks, D. J., Multiple Exciton Generation and Ultrafast Exciton Dynamics in Hgte Colloidal Quantum Dots. PCCP 2013, 15, 16864-16873.

(11) Leontiadou, M. A.; Al-Otaify, A.; Kershaw, S. V.; Zhovtiuk, O.; Kalytchuk, S.; Mott, D.; Maenosono, S.; Rogach, A. L.; Binks, D. J., Ultrafast Exciton Dynamics in Cdxhg(1-X)Te Alloy Quantum Dots. Chem. Phys. 2016, 469, 25-30.

(12) Stolle, C. J.; Schaller, R. D.; Korgel, B. A., Efficient Carrier Multiplication in Colloidal Cuinse2 Nanocrystals. The Journal of Physical Chemistry Letters 2014, 5, 3169-3174.

(13) Cirloganu, C. M.; Padilha, L. A.; Lin, Q.; Makarov, N. S.; Velizhanin, K. A.; Luo, H.; Robel, I.; Pietryga, J. M.; Klimov, V. I., Enhanced Carrier Multiplication in Engineered Quasi-Type-Ii Quantum Dots. Nat Commun 2014, 5, 4148. 
(14) Aerts, M.; Bielewicz, T.; Klinke, C.; Grozema, F. C.; Houtepen, A. J.; Schins, J. M.; Siebbeles, L. D., Highly Efficient Carrier Multiplication in Pbs Nanosheets. Nature Communications 2014, 5, 3789.

(15) Stubbs, S. K.; Hardman, S. J. O.; Graham, D. M.; Spencer, B. F.; Flavell, W. R.; Glarvey, P.; Masala, O.; Pickett, N. L.; Binks, D. J., Efficient Carrier Multiplication in Inp Nanoparticles. Physical Review B 2010, 81, 081303.

(16) McElroy, N.; Page, R. C.; Espinbarro-Valazquez, D.; Lewis, E.; Haigh, S.; O'Brien, P.; Binks, D. J., Comparison of Solar Cells Sensitised by Cdte/Cdse and Cdse/Cdte Core/Shell Colloidal Quantum Dots with and without a Cds Outer Layer. Thin Solid Films 2014, 560, 65-70.

(17) Gachet, D.; Avidan, A.; Pinkas, I.; Oron, D., An Upper Bound to Carrier Multiplication Efficiency in Type Ii Colloidal Quantum Dots. Nano Lett. 2010, 10, 164-170.

(18) Dennis, A. M.; Mangum, B. D.; Piryatinski, A.; Park, Y. S.; Hannah, D. C.; Casson, J. L.; Williams, D. J.; Schaller, R. D.; Htoon, H.; Hollingsworth, J. A., Suppressed Blinking and Auger Recombination in near-Infrared Type-Ii Inp/Cds Nanocrystal Quantum Dots. Nano Lett. 2012, 12, 5545-5551.

(19) Lide, D. R., CRC Handbook of Chemistry and Physics, 90th Ed.; CRC Press: Boca Raton, FL, 2009.

(20) Wu, K. F.; Song, N. H.; Liu, Z.; Zhu, H. M.; Rodriguez-Cordoba, W.; Lian, T. Q., Interfacial Charge Separation and Recombination in Inp and Quasi-Type Ii Inp/Cds Core/Shell Quantum Dot-Molecular Acceptor Complexes. J. Phys. Chem. A 2013, 117, 7561-7570.

(21) Leontiadou, M. A.; Tyrrell, E. J.; Smith, C. T.; Espinobarro-Velazquez, D.; Page, R.; O'Brien, P.; Miloszewski, J.; Walsh, T.; Binks, D.; Tomić, S., Influence of Elevated Radiative Lifetime on Efficiency of Cdse/Cdte Type Ii Colloidal Quantum Dot Based Solar Cells. Sol. Energy Mater. Sol. Cells 2016.

(22) Smith, C. T.; Tyrrell, E. J.; Leontiadou, M. A.; Miloszewski, J.; Walsh, T.; Cadirci, M.; Page, R.; O'Brien, P.; Binks, D.; Tomić, S., Energy Structure of Cdse/Cdte Type Ii Colloidal Quantum Dots-Do Phonon Bottlenecks Remain for Thick Shells? Sol. Energy Mater. Sol. Cells 2016, 158, 160-167.

(23) Reiss, P.; Protiere, M.; Li, L., Core/Shell Semiconductor Nanocrystals. Small 2009, 5, 154-168.

(24) Califano, M.; Gómez-Campos, F. M., Universal Trapping Mechanism in Semiconductor Nanocrystals. Nano Lett. 2013, 13, 2047-2052.

(25) Smith, C. T.; Leontiadou, M. A.; Page, R.; O'Brien, P.; Binks, D. J., Ultrafast Charge Dynamics in Trap-Free and Surface-Trapping Colloidal Quantum Dots. Advanced Science 2015, 2, 1500088.

(26) The spectral range of the detector used limited photoluminescence measurements to $<800 \mathrm{~nm}$.

(27) Zhu, H.; Song, N.; Rodriguez-Cordoba, W.; Lian, T., Wave Function Engineering for Efficient Extraction of up to Nineteen Electrons from One Cdse/Cds Quasi-Type Ii Quantum Dot. J. Am. Chem. Soc. 2012, 134, 4250-4257.

(28) Li, L.; Reiss, P., One-Pot Synthesis of Highly Luminescent Inp/Zns Nanocrystals without Precursor Injection. J. Am. Chem. Soc. 2008, 130, 11588-+. 
(29) Virieux, H.; Le Troedec, M.; Cros-Gagneux, A.; Ojo, W. S.; Delpech, F.; Nayral, C.; Martinez, H.; Chaudret, B., Inp/Zns Nanocrystals: Coupling Nmr and Xps for Fine Surface and Interface Description. J. Am. Chem. Soc. 2012, 134, 19701-19708.

(30) Spencer, B. F., et al., Dynamics in Next-Generation Solar Cells: Time-Resolved Surface Photovoltage Measurements of Quantum Dots Chemically Linked to Zno (10(1)overBar0). Faraday Discuss. 2014, 171, 275-298.

(31) Cant, D. J. H., et al., Surface Properties of Nanocrystalline Pbs Films Deposited at the Water-Oil Interface: A Study of Atmospheric Aging. Langmuir 2015, 31, 1445-1453.

(32) Chen, S. G.; Paulose, M.; Ruan, C.; Mor, G. K.; Varghese, O. K.; Kouzoudis, D.; Grimes, C. A., Electrochemically Synthesized Cds Nanoparticle-Modified Tio2 Nanotube-Array Photoelectrodes: Preparation, Characterization, and Application to Photoelectrochemical Cells. Journal of Photochemistry and Photobiology a-Chemistry 2006, 177, 177-184.

(33) Shard, A. G., A Straightforward Method for Interpreting Xps Data from Core-Shell Nanoparticles. J Phys Chem C 2012, 116, 16806-16813.

(34) Tanuma, S.; Powell, C. J.; Penn, D. R., Calculation of Electron Inelastic Mean Free Paths (Imfps) Vii. Reliability of the Tpp-2m Imfp Predictive Equation. Surf. Interface Anal. 2003, 35, 268-275.

(35) Powell, C. J.; Jablonski, A., Nist Electron Inelastic Mean Free Path Database. Technology, N. I. o. S. a., Ed. Gaithersburg, 2010.

(36) Tanuma, S.; Powell, C. J.; Penn, D. R., Calculations of Electron Inelastic Mean Free Paths .5. Data for 14 Organic-Compounds over the 50-2000 Ev Range. Surf. Interface Anal. 1994, 21, 165-176.

(37) Hernández-Calderón, I., Optical Properties and Electronic Structure of Wide Band Gap Ii-Vi Semiconductors. In Ii-Vi Semiconductor Materials and Their Applications, Taylor and Francis: NEW yORK, 2002; pp 113-170.

(38) Klimov, V. I., Spectral and Dynamical Properties of Multilexcitons in Semiconductor Nanocrystals. In Annu. Rev. Phys. Chem., 2007; Vol. 58, pp 635-673.

(39) McGuire, J. A.; Joo, J.; Pietryga, J. M.; Schaller, R. D.; Klimov, V. I., New Aspects of Carrier Multiplication in Semiconductor Nanocrystals. Acc. Chem. Res. 2008, 41, 18101819.

(40) McGuire, J. A.; Sykora, M.; Joo, J.; Pietryga, J. M.; Klimov, V. I., Apparent Versus True Carrier Multiplication Yields in Semiconductor Nanocrystals. Nano Lett. 2010, 10, 2049-2057.

(41) Midgett, A. G.; Hillhouse, H. W.; Hughes, B. K.; Nozik, A. J.; Beard, M. C., Flowing Versus Static Conditions for Measuring Multiple Exciton Generation in Pbse Quantum Dots. J Phys Chem C 2010, 114, 17486-17500.

(42) Beard, M. C.; Ellingson, R. J., Multiple Exciton Generation in Semiconductor Nanocrystals: Toward Efficient Solar Energy Conversion. Laser \& Photonics Review 2008, 2, 377-399.

(43) Yeh, J. J., Atomic Calculation of Photoionization Cross-Sections and Asymmetry Parameters; Gordon and Breach Science Publishers: Langhorne, PE, 1993.

(44) Page, R. C., et al., Near-Unity Quantum Yields from Chloride Treated Cdte Colloidal Quantum Dots. Small 2015, 11, 1548-54. 
(45) Espinobarro-Velazquez, D.; Leontiadou, M. A.; Page, R. C.; Califano, M.; O'Brien, P.; Binks, D. J., Effect of Chloride Passivation on Recombination Dynamics in Cdte Colloidal Quantum Dots. Chemphyschem 2015, 16, 1239-1244. 
TOC Graphic:

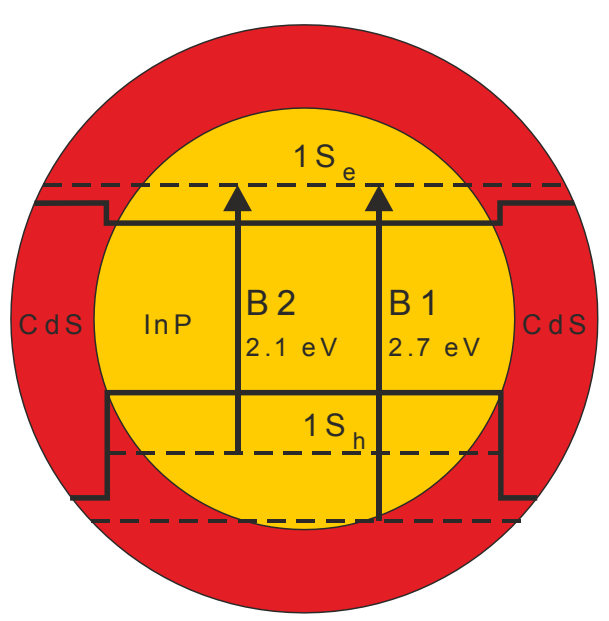

1

2

3

4

5

6
7

8

9

10

11

12

13

14

15

16

17

18

19

20

21

22

23

24

25

26

27

28

29

30

31

32

33

34

35

36

37

38

39

40

41

42

43

44

45

46

47

48

49

50

51

52

53

54

55

56

57

58

59

60 


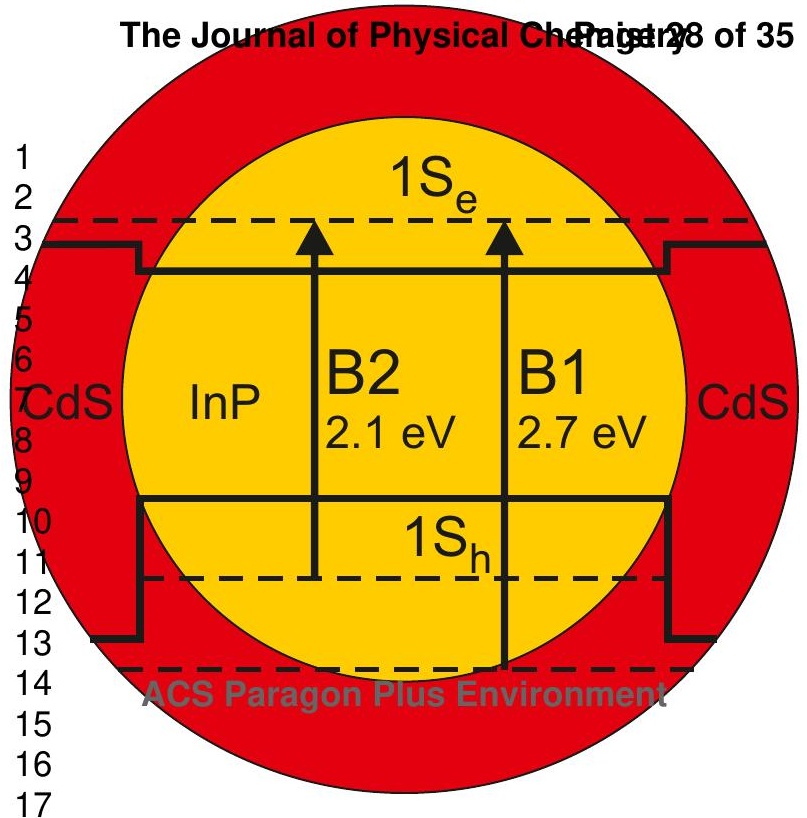




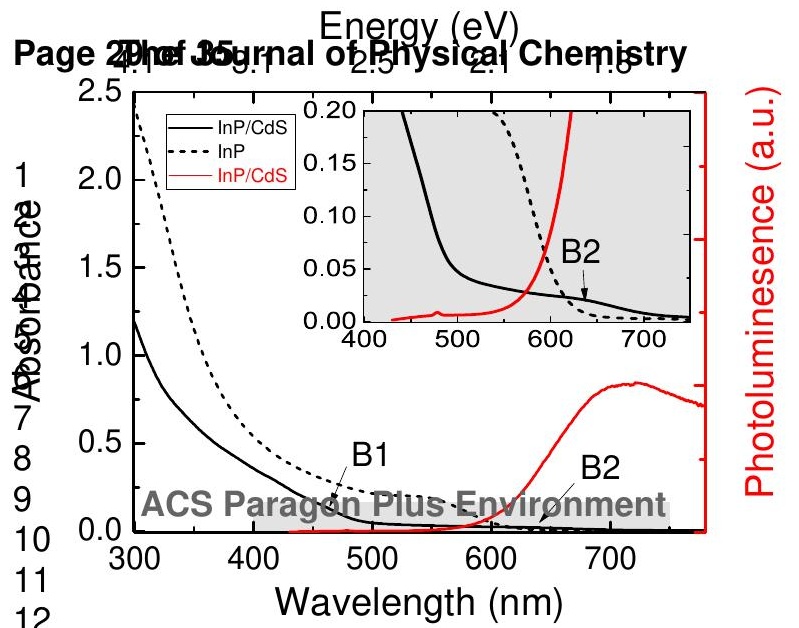




\section{he doumal of Physpat or a.uisty}

13.

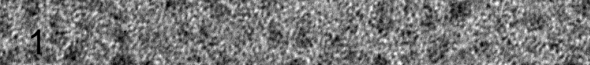

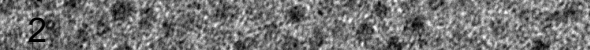
2.

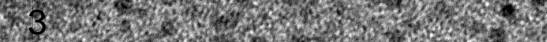

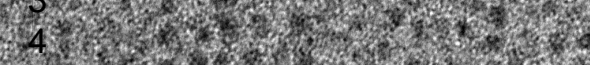

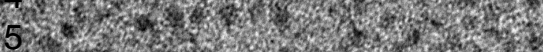
6
$4,7+2$
Wx 2 ,

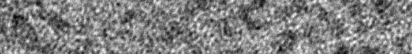
(2).

0.

7

(8.

(f)

9. 10 25:

4. 8989

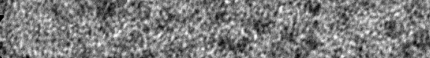

8.0200

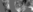

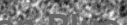

$\rightarrow$

sid

28

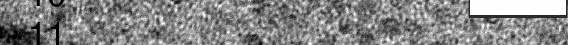
. 20 
Page 3ihof 35urnal of Physical Chemistry

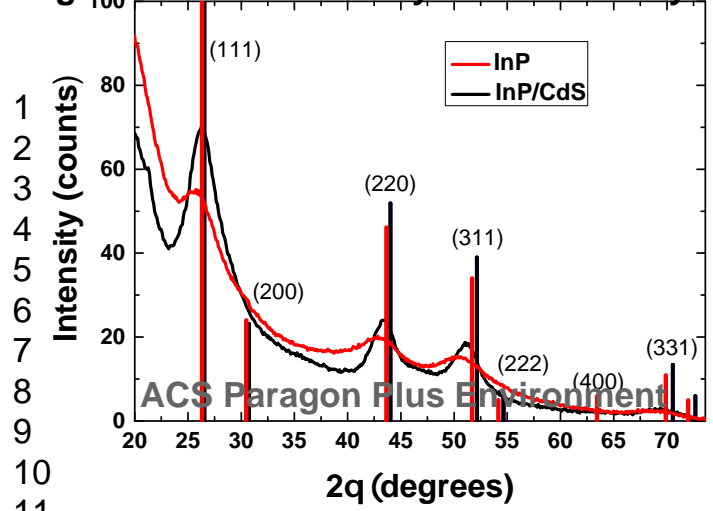




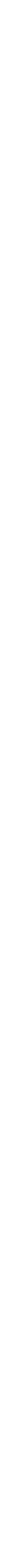




\section{Energy (eV)}

Page 3Bhepesurmat of Physical 1 Chemgstry 1.8

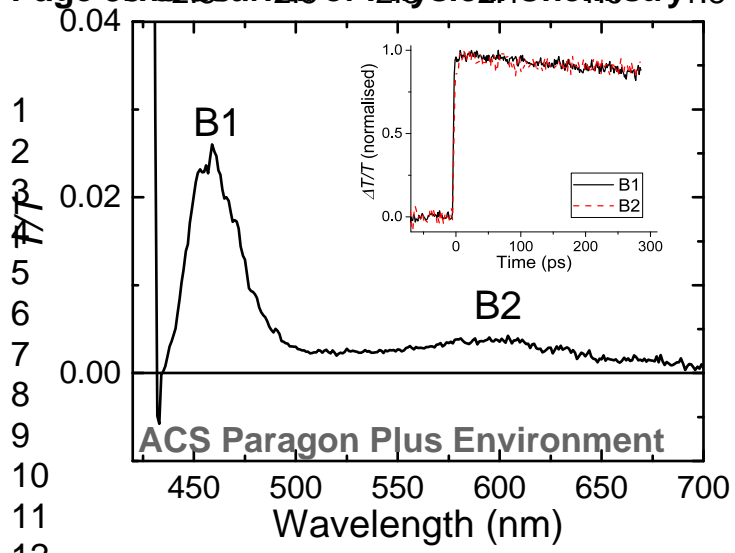




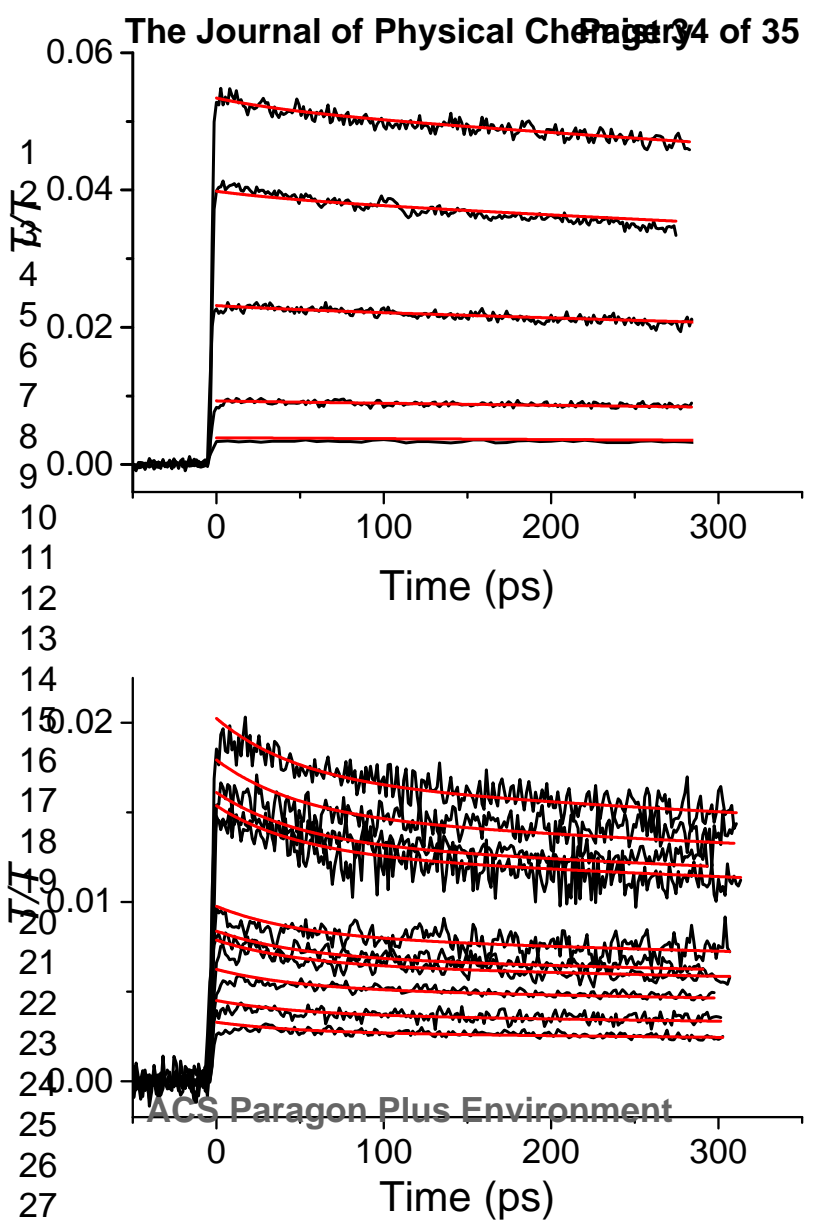


Page 36hef B5urnal of Physical Chemistry

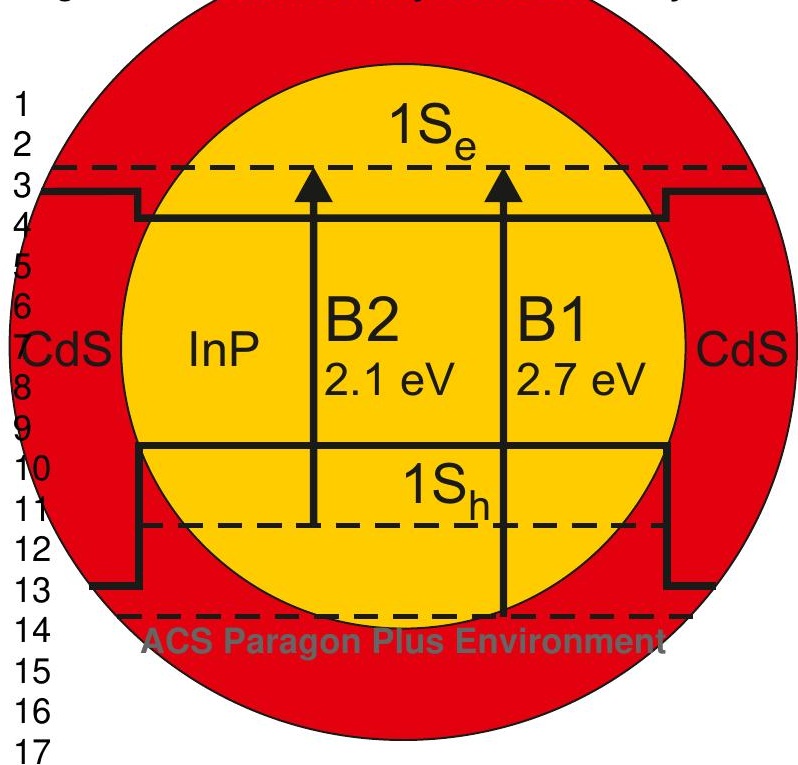

